\title{
Sacroiliitis in Systemic Lupus Erythematosus Revisited
}

 \\ ${ }^{1}$ Department of Internal Medicine, Division of Rheumatology, Demiroğlu Bilim University Medial Faculty, Istanbul, Turkey \\ ${ }^{2}$ Department of Internal Medicine, Division of Rheumatology, Kocaeli University Medial Faculty, Kocaeli, Turkey \\ ${ }^{3}$ Department of Geriatrics, Demiroğlu Bilim University Medial Faculty, İstanbul, Turkey \\ ${ }^{4}$ Department of Radiology, Demiroğlu Bilim University Medial Faculty, Istanbul, Turkey
}

\begin{abstract}
Objectives: This study aims to investigate the prevalence of inflammatory back pain (IBP) and sacroiliitis in a systemic lupus erythematosus (SLE) population as well as the association between IBP and the frequency of human leukocyte antigen B27 (HLA-B27).

Patients and methods: The study included 281 SLE patients ( 16 males, 265 females; mean age 39.9 \pm 11.9 years; range, 20 to 69 years) and 100 healthy controls (HCs) (2 males, 98 females; mean age 41.2+10.1 years; range, 19 to 64 years). Participants were administered a five-item Assessment of SpondyloArthritis international Society-IBP questionnaire. Patients and controls with IBP underwent detailed clinical and laboratory examinations to detect sacroiliitis. Radiographic evaluations were performed by a blinded rheumatologist and radiologist. Interobserver reliability was assessed with Cohen's kappa test.

Results: According to the questionnaire, IBP was present in 46 SLE patients $(46 / 281 ; 16.3 \%$ ) whereas none of the HC had IBP ( $<<0.001$ ). In radiological assessment, 22 SLE patients (7.8\%) had sacroiliitis detected by conventional X-ray and/or magnetic resonance imaging. Only one SLE patient with sacroiliitis had HLA-B27.

Conclusion: Our study showed that IBP is increased in SLE patients and IBP in SLE is not associated with HLA-B27.

Keywords: Inflammatory back pain, lupus, sacroiliitis.
\end{abstract}

Chronic back pain is one of the most common complaints in rheumatology outpatient clinics. Inflammatory back pain (IBP) typically affects patients with spondyloarthropathies (SpA) and has rarely been reported in systemic lupus erythematosus (SLE). Patients with SLE usually suffer from migratory or persistent and polyarticular peripheral arthritis, and about 53 to $95 \%$ of patients have musculoskeletal symptoms. ${ }^{1} \quad$ Nevertheless, tendinopathy (particularly, Achilles and patellar tendon)., ${ }^{2,3}$ and sacroiliitis have rarely been demonstrated on the plain film radiographs and radionuclide bone scans particularly in patients with active lupus. ${ }^{4,5}$
Inflammatory back pain is the key clinical symptom of ankylosing spondylitis (AS), a protype of SpA. Although the etiology is unknown, AS is thought to be associated with CD8 T-cell mediated immunity due to its close relationship with human leukocyte antigen B27 (HLA-B27). ${ }^{6}$ Braun et al. ${ }^{7}$ showed previously that tissue biopsies of sacroiliac (SI) joints were infiltrated by both T cells (CD4 and CD8 positive) and macrophages. Additionally, recent studies demonstrated that autoantibodies may also present in AS patients. ${ }^{8}$ Two mechanisms may be associated with increased autoantibodies in patients with AS. First, excessive production of interleukin (IL)-17 by $\mathrm{T}$ cells ${ }^{9}$ may directly

Received: March 24, 2019 Accepted: August 05, 2019 Published online: January 08, 2020

Correspondence: Neslihan Yılmaz, MD. Demiroğlu Bilim Üniversitesi Tıp Fakültesi İç Hastalıkları Anabilim Dalı Romatoloji Bilim Dalı, 34381 Şişli, İstanbul, Türkiye. Tel: +90 532 - 7425594 e-mail: dr.yilmazneslihan@gmail.com 
stimulate B cell maturation and immunoglobulin production, which was also shown in SLE. ${ }^{10}$ Second, existence of $\mathrm{T}$ and $\mathrm{B}$ cells in inflamed areas may lead to autoimmunity in the presence of appropriate cytokine stimulation. ${ }^{8}$

Systemic lupus erythematosus is characterized by alterations in $\mathrm{T}$ and $\mathrm{B}$ cell functions and results in a production of a variety of autoantibodies. ${ }^{11}$ Increased autoantibody production and increased IL-17 and interferon alpha production are part of complex immune mechanisms that lead to clinical manifestations and organ damage in SLE. Since similar pathogenic mechanisms govern in AS that lead to production of autoantibodies, we hypothesize that sacroiliitis might be increased in SLE. Therefore, in this study, we aimed to investigate the prevalence of IBP and sacroiliitis in a SLE population as well as the association between IBP and the frequency of HLA-B27.

\section{PATIENTS AND METHODS}

The study was conducted at Demiroğlu Bilim University Medial Faculty between June 2010 and June 2015 and included 281 SLE patients (16 males, 265 females; mean age 39.9+11.9 years; range, 20 to 69 years) who fulfilled the 1997 revised American College of Rheumatology criteria and 100 healthy controls (HCs) (2 males, 98 females; mean age $41.2 \pm 10.1$ years; range, 19 to 64 years). All patients and HCs were questioned by face-to-face interviews. The exclusion criteria for the study were age $<18$ years, or presence of lumber disc degeneration or other mechanical pathologies which cause lower back pain. The study protocol was approved by the Demiroğlu Bilim University Medial Faculty Ethics Committee. A written informed consent was obtained from each patient. The study was conducted in accordance with the principles of the Declaration of Helsinki.

Inflammatory back pain was defined according to the Assessment of SpondyloArthritis international Society (ASAS)-IBP criteria. $^{12}$ Therefore, following clinical history items were assessed in a yes/no fashion: (i) age at onset $<40$ years, (ii) insidious onset, (iii) improvement with exercise, (iv) no improvement with rest and (v) pain at night with improvement upon getting out of bed.
All patients and HCs were also questioned for psoriasis, reactive arthritis, uveitis, and family history. Serum autoantibody profiles (antinuclear antibodies, anti-double stranded deoxyribonucleic acid, anti-Smith, antiribonucleoprotein, anti-Sjögren's-syndromerelated antigen A [Anti Ro], anti-Sjögren'ssyndrome-related antigen B [Anti La]) were detected using immuno-blotting technique at the time of the interview. HLA-B27 measurement was also performed by flow cytometry technique.

Standard pelvic radiographs were obtained in all SLE patients with IBP and scored for sacroiliitis according to the modified New York criteria ${ }^{13}$ by a blinded rheumatologist and radiologist. We scored each SI joint as follows: grade 0: normal; grade 1: suspicious; grade 2: minimal abnormality with small localized erosions, sclerosis without joint space alteration; grade 3: definite abnormality with erosion, sclerosis, and joint space widening or narrowing or partial ankylosis; grade 4: total ankylosis of joint.

Magnetic resonance imaging (MRI) of SI joints was ordered in patients with IBP and normal conventional pelvic radiographs. MRI examination was performed with Siemens MAGNETOM Symphony 1.5 Tesla MRI devices (Siemens Medical Systems, Erlangen, Germany), $3 \mathrm{~mm}$ slice thickness. Intravenous administration of gadolinium $(0.1 \mathrm{~mL} / \mathrm{kg}$ dose $)$ was used in all patients. Low signal intensity in $\mathrm{T} 1$ weighted (W) images and high signal intensity in T2W and fats at T2W images were accepted as edema and inflammation (type 1), high signal intensity in both T1W and T2W images and signal loss in fat-saturated T2W images were accepted as fatty degeneration (type 2), low signal intensity in both T1W and T2W images was accepted as sclerotic degenerative changes (type 2). Type 1 changes, enhancement after contrast application, and joint effusion were accepted as acute sacroiliitis, type 2 changes, narrowing or widening in SI joint space and irregularity in joint surface, subchondral bone resorption, ankylosis without type 1 changes or enhancement were accepted criteria for structural changes in MRI evaluation.

\section{Statistical analysis}

Statistical analysis was performed using the Statistical Package for the Social Sciences (SPSS) version 11.5 software (SPSS Inc., 
Table 1. Demographic features of systemic lupus erythematosus patients with inflammatory back pain

\begin{tabular}{|c|c|c|c|}
\hline & \multicolumn{3}{|c|}{ SLE patients with IBP } \\
\hline & $\mathrm{n}$ & $\%$ & Mean \pm SD \\
\hline Age (year) & & & $36.4 \pm 9.9$ \\
\hline $\begin{array}{l}\text { Sex } \\
\quad \text { Female } \\
\text { Male }\end{array}$ & $\begin{array}{c}45 \\
1\end{array}$ & & \\
\hline \multicolumn{4}{|l|}{ Disease manifestations } \\
\hline Joint involvement & 28 & 60.8 & \\
\hline Skin involvement & 20 & 43.4 & \\
\hline Hematologic involvement & 19 & 41.3 & \\
\hline Nephritis & 10 & 21.7 & \\
\hline Neurolupus & 7 & 15.2 & \\
\hline Serositis & 3 & 6.5 & \\
\hline Lung involvement & 1 & 2.1 & \\
\hline \multicolumn{4}{|l|}{ Autoantibodies } \\
\hline Anti-DNA & 25 & 54.3 & \\
\hline Anti-Ro & 10 & 21.7 & \\
\hline Anti-RNP & 8 & 17.3 & \\
\hline Anti-Sm & 4 & 8.6 & \\
\hline Anti-La & 4 & 8.6 & \\
\hline Anti-ribosomal P & 2 & 4.3 & \\
\hline Antiphospholipid syndrome & 8 & 17.3 & \\
\hline \multicolumn{4}{|c|}{$\begin{array}{l}\text { SLE: Systemic lupus erythematosus; IBP: Inflammatory back pain; } \\
\text { SD: Standard deviation; Anti-DNA: Anti-deoxyribonucleic acid; } \\
\text { Anti-Ro: Anti-Sjögren's-syndrome-related antigen A; Anti-RNP: } \\
\text { Anti-ribonucleoprotein; Anti-Sm: Anti-Smith; Anti-La: Anti-Sjögren's- } \\
\text { syndrome-related antigen B. }\end{array}$} \\
\hline
\end{tabular}

Chicago, IL, USA). The Mann-Whitney U test was performed to compare the qualitative variables, while Fisher's exact test was used in quantitative variables. Interobserver reliability was assessed with Cohen's kappa $(\kappa)$ test, with $\kappa$ value $>0.75$ representing good agreement, 0.4-0.75 representing fair to good agreement and $<0.40$ representing poor agreement.

\section{RESULTS}

A total of 281 SLE patients were evaluated and compared with 100 age- and sex-adjusted $\mathrm{HCs}$ at baseline. The mean age were similar between SLE patients and HCs $(39.9 \pm 11.9$ years vs. $41.2 \pm 10.1$ years, respectively; $\mathrm{p}>0.05$ ). The disease duration of SLE patients was 7.4 \pm 5.7 years. Baseline characteristics of SLE-IBP patients are summarized in Table 1.

Of the 281 SLE patients, 46 fulfilled the ASAS criteria for IBP (16.3\%), whereas none of the HCs had IBP (SLE vs. HC, $\mathrm{p}<0.001$ ). Only one SLE patient with IBP had a previous history of uveitis, one patient had family history of spondyloarthritis and one had relatives with Behçet's disease.

Conventional radiographic evaluation of SI joints was performed in all SLE patients with IBP and unilateral or bilateral grade 2 sacroiliitis was detected in six patients. MRI analysis of SI joints was obtained in 31 out of 40 (77.5\%) SLE patients who had IBP, but not radiographic sacroiliitis. Bone marrow edema or contrast enhancement in SI joints (active sacroiliitis) were found in 14 patients, while sclerosis or fatty degeneration (structural changes) were revealed in two patients (Figures 1) in MRI. Good agreement was found by Cohen's $\kappa$ test between the radiologist and rheumatologist on radiological assessment $(\kappa=0.80)$. Only one SLE patient with sacroiliitis was positive for HLA-B27 and she had a history of uveitis.


Figure 1. Contrast-enhanced magnetic resonance imaging of sacroiliac joints. Coronal plane (a) Fat-saturated T2W, (b) Fat-saturated precontrast T1W, (c) Fat-saturated postcontrast T1W images; type 1 signal changes in iliac joint faces and sacroiliac joint space with enhancement accepted as acute sacroiliitis (white arrows). W: Weighted. 


\section{DISCUSSION}

Inflammatory back pain usually starts insidiously in the lumbar region and is often associated with SI inflammation. The prevalence of IBP is estimated to be $4-6 \%$ in the general population. ${ }^{14,15}$ This study, designed to investigate the frequency of IBP and sacroiliitis in SLE, revealed that IBP was increased in lupus patients compared to the healthy population.

Different sets of validated criteria with comparable sensitivity and specificity can be used to detect IBP early. The frequency of IBP in SLE according to these validated criteria has been reported in a few case reports., $4,16,17$ In a recent prospective study from Mexico, the authors evaluated 192 consecutive patients with SLE, and the prevalence of IBP was found as $10 \%$ according to Calin criteria; sacroiliitis of any degree was $16 \%$. Here, we similarly found that $16.3 \%$ of our SLE patients met the ASAS 2010 criteria for IBP; however, sacroiliitis rate $(7.8 \%$ of all SLE patients) was lower than that reported by Sierra-Jimenez et al. ${ }^{18}$

Human leukocyte antigen B27 and spondylitis susceptibility is one of the strongest known HLA-disease associations. The prevalence of HLA-B27 is reported to be $6.8 \%$ in healthy population in Turkey. Therefore, we hypothesized that IBP and sacroiliitis might be increased in SLE as a result of high prevalence of HLA-B27. However, we noted HLA-B27 positivity only in one patient in our cohort. Nassonova et al. ${ }^{19}$ similarly reported that male lupus patients who had radiologic evidence of sacroiliitis were HLA-B27 negative. This finding implies that factors other than HLA-B27 may play a role in existence of sacroiliitis in SLE patients.

In recent years, IL-23/IL-17 pathway has been shown to have significance in the pathogenesis of $\mathrm{AS}^{20}$ Although SLE is suggested to be a T helper type 2 (Th2) cell driven disease, recently, numerous trials showed increased IL-17 levels in SLE patients. ${ }^{21}$ Besides proinflammatory activity, new evidence suggests that aberrant production of IL-17 may drive pathogenic antibody response through its ability to induce ectopic germinal centers in autoimmune disease ${ }^{22}$ and participate in the activation of $\mathrm{B}$ cells in SLE. ${ }^{23}$ Taken together, IL-17 and Th17 cells play important roles in autoimmunity. In some trials, IL-17producing $T$ cells have been shown in the kidney of patients with SLE nephritis and increased local levels of IL-17 have also been found in skin and central nervous system lesions. ${ }^{17}$ We can speculate that IL-17 may be the pathogenic link between sacroiliitis and SLE which needs further investigation.

This study has some limitations. Firstly, due to our hospital-based approach, there could be a potential bias for more severe lupus cases being included in the study. We tried to avoid this bias recruiting consecutive patients; however, still, our results may not reflect the true prevalence. Secondly, we could not perform axial imaging in all patients due to restrictions by the Ethics Committee. And lastly, even though we think that IL-17 is the main link between sacroiliitis and SLE, we could not manage to study IL-17 at the same time in a larger cohort.

In conclusion, IBP is not rare in SLE patients; however, most patients had no severe radiological evidence of sacroiliitis suggesting a mild axial disease. IBP in SLE is not associated with HLA-B27.

\section{Declaration of conflicting interests}

The authors declared no conflicts of interest with respect to the authorship and/or publication of this article.

\section{Funding}

The authors received no financial support for the research and/or authorship of this article.

\section{REFERENCES}

1. Labowitz R, Schumacher HR Jr. Articular manifestations of systemic lupus erythematosus. Ann Intern Med 1971;74:911-21.

2. Papanikolaou A, Charalambides C, Thanassas C. Letter to the Editor. Spontaneous simultaneous bilateral patellar tendon rupture in a systemic lupus erythematosus patient. Lupus 2007;16:915-7.

3. Aydin S, Atagunduz P, Filippucci E, Yavuz $\mathrm{S}$, Direskeneli $\mathrm{H}$. A rare case of spontaneous, bilateral Achilles tendon rupture in systemic lupus erythematosus and a review of the literature. Lupus 2008; 17:1051-2.

4. De Smet AA, Mahmood T, Robinson RG, Lindsley HB. Elevated sacroiliac joint uptake ratios in systemic lupus 
erythematosus. AJR Am J Roentgenol 1984;143:351-4.

5. Lee SS. Symptomatic unilateral sacroiliitis in systemic lupus erythematosus. Lupus 1995;4:328-9.

6. Benjamin R, Parham P. Guilt by association: HLAB27 and ankylosing spondylitis. Immunol Today 1990;11:137-42.

7. Braun J, Bollow M, Neure L, Seipelt E, Seyrekbasan $\mathrm{F}$, Herbst $\mathrm{H}$, et al. Use of immunohistologic and in situ hybridization techniques in the examination of sacroiliac joint biopsy specimens from patients with ankylosing spondylitis. Arthritis Rheum 1995;38:499-505.

8. Wright C, Sibani S, Trudgian D, Fischer R, Kessler B, LaBaer J, et al. Detection of multiple autoantibodies in patients with ankylosing spondylitis using nucleic acid programmable protein arrays. Mol Cell Proteomics 2012;11:00384.

9. Shen H, Goodall JC, Hill Gaston JS. Frequency and phenotype of peripheral blood Th17 cells in ankylosing spondylitis and rheumatoid arthritis. Arthritis Rheum 2009;60:1647-56.

10. Doreau A, Belot A, Bastid J, Riche B, TrescolBiemont MC, Ranchin B, et al. Interleukin 17 acts in synergy with B cell-activating factor to influence B cell biology and the pathophysiology of systemic lupus erythematosus. Nat Immunol 2009;10:778-85.

11. Crispin JC, Tsokos GC. Pathogenesis of lupus. In: Hochberg MC, editor. Rheumatology. Vol 2. 5th ed. Philadelphia: Elsevier; Section 2011. p. 1289-93.

12. Rudwaleit M, Metter A, Listing J, Sieper J, Braun J. Inflammatory back pain in ankylosing spondylitis: a reassessment of the clinical history for application as classification and diagnostic criteria. Arthritis Rheum 2006;54:569-78.

13. van der Linden S, Valkenburg HA, Cats A. Evaluation of diagnostic criteria for ankylosing spondylitis. A proposal for modification of the New York criteria. Arthritis Rheum 1984;27:361-8.

14. Hoy D, Bain C, Williams G, March L, Brooks P, Blyth
$\mathrm{F}$, et al. A systematic review of the global prevalence of low back pain. Arthritis Rheum 2012;64:2028-37.

15. Reveille JD, Weisman MH. The epidemiology of back pain, axial spondyloarthritis and HLA-B27 in the United States. Am J Med Sci 2013;345:431-6.

16. Kohli M, Bennett RM. Sacroiliitis in systemic lupus erythematosus. J Rheumatol 1994;21:170-1.

17. Chandrasekhara PK, Jayachandran NV, Thomas J, Narsimulu G. Systemic lupus erythematosus and dermatomyositis with symptomatic bilateral sacroiliitis: an unusual and interesting association. Mod Rheumatol 2009;19:84-6.

18. Sierra-Jimenez G, Sanchez-Ortiz A, Aceves-Avila FJ, Hernandez-Rios G, Durán-Barragán S, RamosRemus C. Tendinous and ligamentous derangements in systemic lupus erythematosus. $\mathrm{J}$ Rheumatol 2008;35:2187-91.

19. Nassonova VA, Alekberova ZS, Folomeyev MY, Mylov NM. Sacroiliitis in male systemic lupus erythematosus. Scand J Rheumatol 1984;52:23-9.

20. Kenna TJ, Davidson SI, Duan R, Bradbury LA, McFarlane J, Smith M, et al. Enrichment of circulating interleukin-17-secreting interleukin-23 receptorpositive $\gamma / \delta$ T cells in patients with active ankylosing spondylitis. Arthritis Rheum 2012;64:1420-9.

21. Li D, Guo B, Wu H, Tan L, Chang C, Lu Q. Interleukin-17 in systemic lupus erythematosus: $A$ comprehensive review. Autoimmunity 2015;48:353-61.

22. Sweet RA, Lee SK, Vinuesa CG. Developing connections amongst key cytokines and dysregulated germinal centers in autoimmunity. Curr Opin Immunol 2012;24:658-64.

23. Jacob N, Yang H, Pricop L, Liu Y, Gao X, Zheng SG, et al. Accelerated pathological and clinical nephritis in systemic lupus erythematosus-prone New Zealand Mixed 2328 mice doubly deficient in TNF receptor 1 and TNF receptor 2 via a Th17-associated pathway. J Immunol 2009;182:2532-41. 